\title{
Multimodal profusion in the literacies of the Massive Open Online Course
}

\author{
Jeremy Knox* and Siân Bayne \\ Institute of Education, Community and Society, University of Edinburgh, Edinburgh, UK
}

(Received 14 May 2013; final version received 20 August 2013)

This paper takes a view of digital literacy, which moves beyond a focus on technical methods and skills in an attempt to maintain a broader approach that encompasses a critical view of the learning subject. In doing this, we consider socio-materialism and its relation to aspects of literacy theory. We anchor the discussion in a consideration of the 'E-learning and Digital Cultures' Coursera MOOC, which provided a tangible setting for theorising some of the practices of digital literacy differently. The profusion of multimodal artefacts produced in response to this course constituted a complex series of socio-material entanglements, in which human beings and technologies each played a constituent part. Two specific digital artefacts are analysed according to these terms. We conclude that socio-material multimodality constitutes a different way of thinking about digital literacy: not as representational practices, but rather as multifaceted and relational enactments of knowledge, specific to particular contexts and moments.

Keywords: digital literacy; multimodal; socio-material; MOOC; enactment

\section{Introduction}

Digital literacy is the latest in a succession of technological tags and catch-all phrases proposed to encapsulate the educational response to computer systems (for an extensive list see Goodfellow 2011, and for a discussion of origin see Bawden 2008). While there is no all-encompassing definition of digital literacy (Oliver 2012), Goodfellow and Lea (2007) usefully emphasise the distinction between 'digital literacy' as a set of skills or competencies located 'within' an individual, and 'digital literacies' as a more complex engagement with a shifting, volatile 'range of socially and culturally situated practices' (p. 5). Our use of the term in this paper has more in common with the plural usage, though the emphasis here encompasses the sociocultural alongside, and in intimate relation with, the material. Digital literacies sit within a field which often assumes that inevitable changes are sweeping both educational institutions and the wider society; changes that are signalled by the increasing ubiquity of digital networks in particular.

Where digital literacy is understood as a set of identifiable skills it is often related to future projections of social and academic life. Competencies such as searching and managing information, aggregating and re-using existing resources, and expressing ideas with multiple forms of media are proposed, to which students, employees and

*Corresponding author. Email: j.k.knox@sms.ed.ac.uk 


\section{J. Knox and S. Bayne}

citizens progress though training, while having no share in deciding what those future competencies might be (Beetham, McGill, and Littlejohn 2009). JISC situate digital literacy training as an essential requirement for potential employment; skills not necessarily required for an individual's current situation, rather as an investment in a future working self (for example JISC 2013). These competency-based understandings frame the individual as in deficit; demanding the acquiring of digital abilities in order to remain within societal constructions of 'competent'; such a critique aligns with a wider concern about the transformation of higher education into a sector subservient to the economic forces of a neo-liberal political philosophy. It is habitually the responsibility of the individual for becoming digitally literate which is foregrounded, where wider political and economic factors which shape such independence are often neglected.

Elsewhere, digital literacy is framed as essential for the contemporary present. Such 'literacies' are not only perceived as central to an individual's ability to function in society, but also as bestowing 'social 'normality' on its owner' (Goodfellow 2011, p. 131). Such 'normal' contemporary living might involve the use of social media, often considered to be increasingly important to the ways that people communicate across the 'developed' world. Where the technology is supposed to be ever more pervasive, the standard model of human being must be furnished with the appropriate digital dexterity. From this perspective, being digitally literate is no longer a supplement, but a core requirement and a matter of survival.

Broader approaches within the socio-culturally oriented 'digital literacies' emphasise social meaning-making practices with technology. Lankshear and Knobel (2008) call, not for generic aptitudes, but multiple, various and adaptable digital proficiencies. The focus here is on the ability to create, interpret and communicate meaning within cultural contexts, rather than simply formulating lists of definitive skills. Such approaches have been suggested to adopt a 'socio-cultural framing' of literacy, which serves to blur the specificity of the term (Goodfellow 2011). Goodfellow (2011) highlights this as a problematic merging and homogenising of both terms in 'digital literacy', such that technical and cultural sensitivities and distinctions are removed. The disciplines of ICT, computer science, communication and media studies are conflated into an all-encompassing 'digital', over which students are compelled to gain mastery. This calls into question the use of such a catchall label, given the range of diverse community and institutional stakeholders, and the array of technological infrastructures, protocols and codes that impose themselves upon the given task (Goodfellow 2011).

Narratives that situate 'digital literacy' skills as indispensable, justified by projections of far-reaching societal change, are surprisingly pervasive. Ulmer (2003) proposes 'electracy' as an exploration of how we might use internet spaces to work towards a new social and epistemological formation which belongs to the digital age. As Ulmer (2003) says, 'electrate peoples who experience thought as virtual image will organize collectively in some new way that has not come fully into view' (p. 8). While 'electracy' maintains a distinction between the skills required to engage with printed text and those necessary to maximise the promise of digital media, 'transliteracy' has sought a broad definition of literacy to encompass multiple modes and communicative practices, in which digital technology is merely one constituent part (Thomas et al. 2007). Transliteracy is the call for a blurring of distinctions between print-based and digital media, positing instead a 'unifying ecology' of communicative and interpretive skills (Thomas et al. 2007, no page). However, this aptitude appears to be 
located as an innate human quality; a universal facility for engaging with mediated communication, anterior to and distinct from both the information and the means. Furthermore, the non-linear historical approach of transliteracy appears to situate the activities of communication exclusively between human beings, with technology serving as medium and instrument. The notions of acceleration and amplification of communicative capacity tend to suggest intuitive, essential and universal human qualities, just waiting to be improved by successive technological innovations.

While this overview of literature on digital literacies is highly selective, it is intended to highlight a prevailing focus on competencies that are obligatory for present and future societal participation. Whether levelled at the individual, institutions, or society as whole, the ability to communicate with, create meaning from, and interpret the multimodal characteristics of digital media appears mandatory. However, we suggest that these general orientations tend to situate technology in an instrumental role, as the (more-or-less) neutral means of achieving pre-defined educational goals (Friesen and Hamilton 2010). Crucially, these formulations of digital literacy preserve an epistemological orientation that separates us from the technologies we use and the material world around us. The next section will outline socio-materialism, and propose it as an alternative basis for the discussion of digital literacy.

\section{Digital literacy and the socio-material}

The 'socio-material' encompasses a broad range of theoretical approaches to the relationships between the social (human) and the material (non-human). Thus, it entails a decentring of the subject and a challenge to many established educational orthodoxies (Fenwick et al. 2011; Fenwick and Landri 2012), and much of the discourse around digital literacy and competence. It derives from post-foundational philosophy in perceiving objects, properties and boundaries to be enacted by assemblages of human and non-human relations, rather than being anterior to these. Socio-material theory is often associated with a 'materialist turn' which attempts to counter what is perceived to be an over-emphasis on the socially constructed and discursive in explanations of social entities and organisations. In this discussion of education in the digital - a domain often construed as 'virtual' and so 'immaterial' - we interpret the material to be those facets of the software, infrastructure and algorithms of the web that can be understood as acting in a way which is not simply about the 'intention' of their human designers or users. This does not mean that we consider them to be entirely autonomous, but rather as non-human elements continuous with and always related to the human.

Specifically in relation to education, the 'socio-material' is suggested by Fenwick, Edwards, and Sawchuk (2011) as an over-arching term for a range of approaches applicable to educational research and practice: complexity theory, Actor-Network Theory, Cultural Historical Activity Theory and spatiality theories. While marked by particular distinctions, these theoretical areas are suggested to converge on the concern for 'conceptualizing knowledge, capabilities and subjectivities as emerging simultaneously in webs of interconnections among heterogeneous entities: human and non-human, social discourses, activities and meanings, as well as material forces, assemblages, and transformations' (Fenwick et al. 2011, p. 2). It is not our intention here to focus on any one of these specific approaches, but rather to explore instances of this broad orientation toward human and non-human agency and assemblage, something we suggest is under-represented in the digital literacy literature. Neither 


\section{J. Knox and S. Bayne}

do we perceive socio-material approaches to be an 'all-seeing eye' for research. As Mutch (2013) cautions, a tangible grasp of the material is often difficult and 'social structures are not necessarily transparent' (p. 32).

Significantly, socio-material theory is described as an interest in the situatedness of educational processes and the relations between them, rather than with individual teachers, students, or technologies (Fenwick and Landri 2012). Thus, it may provide productive alternatives to work in digital literacies, which tends to perceive context in predominantly cultural terms (Lanshear and Knobel 2008). Socio-material theory suggests that learning is embedded in action and emerges through practice, processes that produce the objects and characteristics of educational events. Fenwick et al. (2011) draw upon much more established traditions of educational theory to claim the ontological indistinguishability of practice and learning in socio-material perspectives. However, their purpose is to foreground the role of the material in analysing educational practices (Fenwick et al. 2011). Educational spaces and objects, in this view, are not considered to be merely backdrops to learning activities, or tools with which to improve educational performance. Rather they are the emergent results of enmeshed social and material enactments. Thus, Fenwick et al. (2011) distinguish their relational socio-materiality from more established theoretical areas which, while taking the material into account (e.g. classrooms, buildings, objects), maintain the separation of the human and non-human (the classroom as a 'container' for educational practices, for example). The view of the material as a set of 'mere instruments to advance educational performance' (Fenwick et al. 2011, p. 1) reflects many of the considerations of technology in the digital literacy literature. As an alternative, we propose here an approach which takes into account coemergence, interdependence and mutual constitution in the consideration of multimodal practices and digital literacy. From this perspective all roles, identities or characteristics are performed by the socio-material assemblage, rather than preexisting it suggesting, significantly, a blurring of the focus on human intention (Fenwick et al. 2011; Hannon 2013). We draw upon socio-material theory therefore, as a challenge to the essential human subject routinely preserved and privileged in the field of digital literacies. Rather than remaining within an ontological structure that maintains an authentic, rational learner contained within the sanctity of the corporeal, a socio-material approach will allow us to explore the hybrid connections and embodied possibilities of educational practices in and among the digital.

Socio-materialism, we suggest, may be a productive way to engage with literacy perspectives that encompass notions of complexity and relationality. Goodfellow and Lea (2009) discuss the hybridity of literacy practices in various institutions; they draw our attention to multiple modes of engaging with resources, both conventionally onand offline, and the intermingling of various hardware and software. Transliteracy, in turn, posits that a range of analogue, digital, historical, cultural and contextual factors shape how an individual interprets a learning task, as well as what kind of work is produced in response. As such a contingent 'lifeworld' is proposed as a 'physical environment and subjective experience' (Thomas et al. 2007, no page), which influences communication and interpretation. Such relations might usefully connect with the socio-material concern for assemblages, networks, and spatial orderings, rather than remaining focussed on the experience of the subject and its essential human characteristics. Descriptions of specific technologies hint at possible alternative readings: 'a Flickr image is understood not as an isolated event but in conjunction with the user's knowledge about what a Flickr page is; what prompted 
that person to post it, and why 16 people left comments. It's not just a photo collecting technology' (Thomas et al. 2007, no page). Thus, while a sense of distributed agency is detectable here, the focus appears to be solely on the intentions and interactions of human beings, disregarding the website itself and the various technologies involved in producing, uploading and accessing the image. Occasionally a more expansive notion of relational subjectivity surfaces: 'Transliteracy happens in the places where different things meet, mix, and rub together. It is an interstitial space teeming with diverse life-forms, languages in many voices, many kinds of scripts and media' (Thomas et al. 2007, no page) and '[t]he 'patterned ways' of transliteracy are multiple, varied and often physical. A sense of how it feels to hold a feather quill, chisel stone, type on a keyboard, or take a photograph, is important and helps connect the material product ... to the means of production' (Thomas et al. 2007, no page). While the material features here, these actions are framed entirely in terms of skills acquisition, and the differing 'tools' seem to be transparent instruments in the pursuit of a universal social communication. We therefore want to suggest that approaches such as transliteracy can be developed to include critical perspectives on technology and the subject so that multimodal practices can be viewed as sociomaterial enactments. It is not just graphocentrism that can be destabilised in the theorisation of digital literacy, but anthropocentrism as well.

In the next section, we suggest that the profusion of multimodal artefacts produced within a particular context - the E-learning and Digital Cultures MOOC provides a tangible and profound opportunity to theorise digital literacies differently using ideas drawn from the socio-material. Our analysis will focus on the capacity to decentre the human as the sole agential force by acknowledging and bringing to the fore relations that connect and hybridise educational enactments in the digital. The key socio-material dimensions of this analysis will be the continuity of materiality and representation; the ways that relations alter connecting elements; and how these processes of hybridity perform particular knowledges and subjectivities.

\section{The E-learning and digital cultures MOOC}

'E-learning and Digital Cultures' was a Massive Open Online Course (MOOC) that took place in January 2013 and became known by the acronym EDCMOOC. A total of 42,000 participants enrolled on this course, which was developed and taught by a team of teachers and researchers from the University of Edinburgh's fully online MSc in Digital Education, and delivered on the Coursera platform. The course sought to explore the intersection of digital and learning cultures online, and comprised a 5-week programme of public domain videos and open access literature from cultural studies and education. While formally a Coursera offering, the EDCMOOC strategy was to encourage participant activity outside of the central platform. There were neither predefined course spaces nor limits to the kind of web services one might use to participate, beyond the initial suggestions of Facebook and Twitter and the recommendation that work be publicly visible. This approach served to create a distributed and emergent course space in which diverse activity and multimodal production could take place.

The lack of formal course structure and the ambiguity of predefined course activities compelled many participants to respond by organising their own methods and strategies for involvement. The act of participation became the 'making sense' of being in digital space. This manifested as a profusion of multimodal artefacts, 


\section{J. Knox and S. Bayne}

produced before the official start date, throughout the duration of the course, and in response to the final assignment. To be digitally literate in the EDCMOOC became a matter of responding to a distributed and relatively amorphous educational space, and using digital media to enact a sense of participation. The term 'digital artefact,' while being the specific term used for the final assignment in the EDCMOOC, will be used subsequently to refer to any and all multimodal student-produced work created in response to the course.

Digital literacy has been described as a blurring of academic and cultural knowledge (Carpenter 2009); it is our suggestion that MOOCs sit precisely at this juncture of the institutional and informal, providing a fertile space for the theorisation of educational, social and technological intersections. However, we suggest the scale of participation in the EDCMOOC disrupted further boundaries. The unprecedented rate, magnitude and localised intensity of multimodal production shifts attention away from the instrumental capabilities of the individual, or the affordances of isolated technologies, and brings to the fore the complex, relational and fluid entanglements of socio-material practices. The following will discuss the profusion of digitality and multimodality in the EDCMOOC, and provide a focussed analysis of two specific artefacts.

Figure 1 depicts a section of the 'EDCMOOC Digital Artefact' Wallwisher, a message based collaborative space that came to accommodate 331 depictions of digital artefacts from the course. While this web page exposed only a small proportion of the 1719 final assignments submitted, and a fraction of the digital work created throughout the duration of the course, it is illustrative of, we suggest,

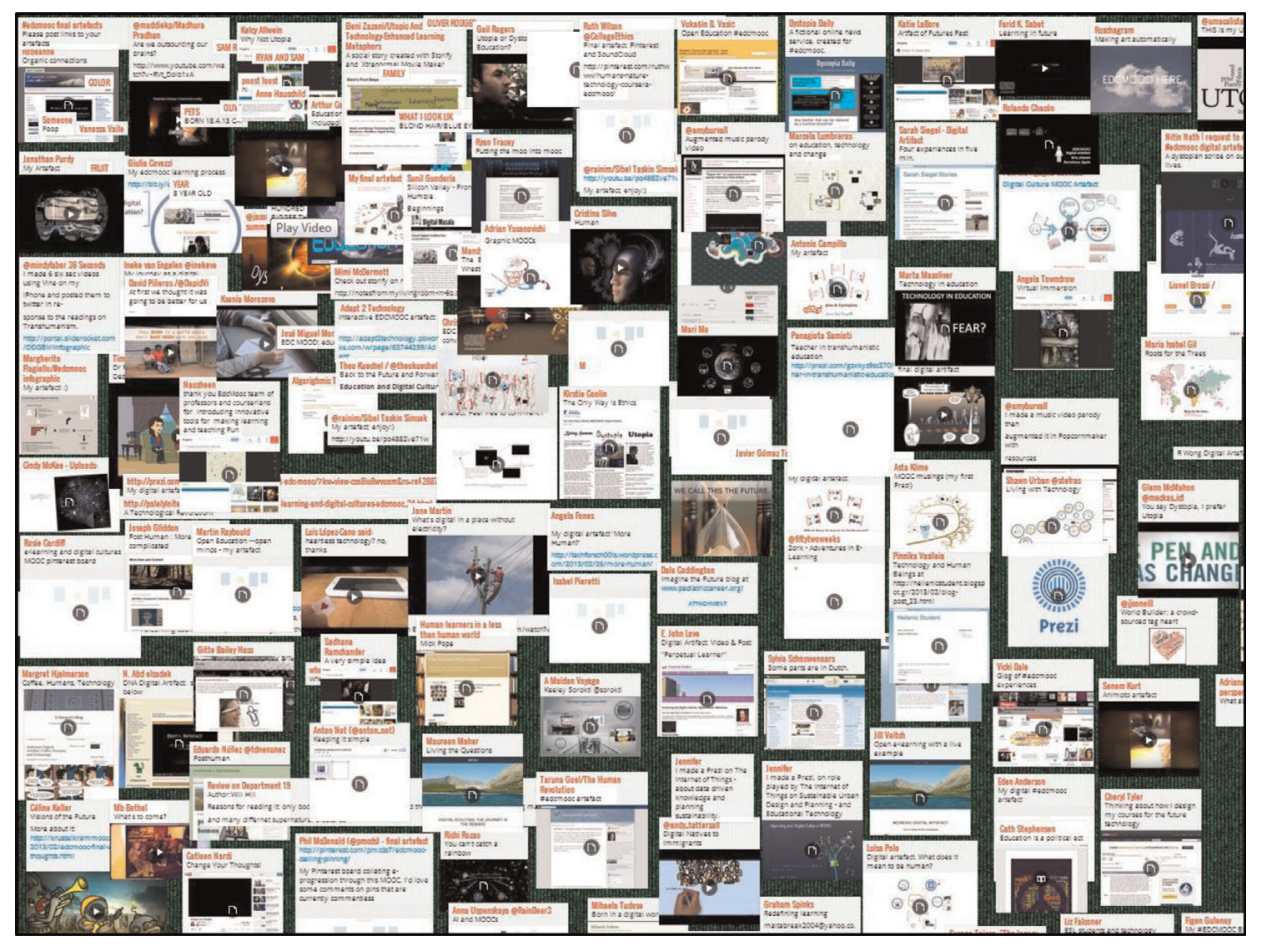

Figure 1. A section of the EDCMOOC digital artefact Wallwisher, illustrating the profusion of multimodal enactments http://padlet.com/wall/edcmooc_artefact 
a profusion of multimodality unprecedented in typical educational scenarios. The experience of encountering such an abundance of multimodal work (experienced by all who participated, both instructors and students alike) had the consequence of shifting focus away from any one producer and towards an intricate and relational socio-material mix. Many participants chose to use the same services, such as You Tube, Xtranormal, Prezi, ThingLink and Videoscribe. Thus, multiple artefacts were created using the same service, and while the individual use was distinct, the repetition of technical functions remained the same, serving to highlight preprogrammed effects; those features of the multimodal that are algorithmic, material, and non-human. This prompts a more in-depth analysis of specific digital artefacts.

The EDCMOOC final assignment submission 'World Builder: a crowd-sourced tag heart' (Figure 2) is illustrative of the intricate assemblages involved in multimodal digital work. The piece is a 'tag-' or 'word-cloud'; a visual representation of text in which single words are displayed in various font sizes according to the frequency with which they occur, and the overall collection arranged to resemble a familiar shape. While it appears as a finalised, stable and contained image, various activities and processes have come together to produce it, involving a number of EDCMOOC participants and a range of web spaces and services. The following analysis is specific to the digital artefact in Figure 2; however, similar word-clouds were created throughout the EDCMOOC, and the claims made here for socio-material complexity can be attributed to much broader course activity.

The text used for this digital artefact was produced in response to one of the video resources used in the course. 'World Builder' (Branit 2007) is a short sci-fi film, depicting a male character that creates an idyllic computer generated holographic environment for the cognitive enjoyment of an apparently comatose female. It is a film which features themes of simulation, immersion in technology, virtuality, and artifice, and these were the interpretations proposed by the course tutors alongside an

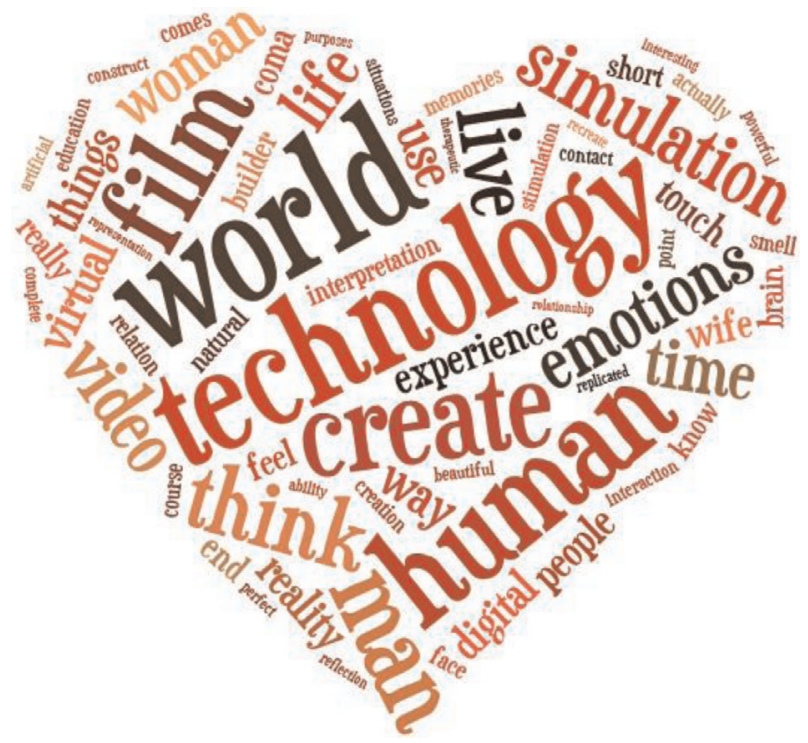

Figure 2. 'World Builder: a crowd-sourced tag heart' by John O'Neill, digital artefact created for the EDCMOOC: http://www.tagxedo.com/artful/f2c519c2502249f9 


\section{J. Knox and S. Bayne}

embedded instance of the video within the Coursera site. If we are to identify a starting point or source for the 'World Builder: a crowd-sourced tag heart,' it is perhaps this mix of (dialogue-less) video and the textual interpretation provided within Coursera. However, origins are perhaps not so simple to ascertain, as the video is simultaneously located on the video-sharing site You Tube, where it is situated in an entirely different web space. Here it is flanked by 'recommended videos' determined by algorithms that measure both meta-data and user activity (Davidson et al. 2010), and buttressed with thousands of public comments which provide alternative interpretations.

The discussion inspired by these spaces took place within a Coursera forum thread, comprised of 85 separate posts and replies from 73 identified and 2 anonymous posters, and it was from this body of text that the word-cloud was derived. A number of these posts quote or refer directly to the interpretations and questions posed by the course tutors; text which not only informed and shaped the subsequent discussion, but also fed directly into the word-cloud generator. The body of text is thus irreducible to a single intention or agency, and derives rather from a distributed array of participants. The gamut of contingencies responsible for the 'World Builder: a crowd-sourced tag heart' are not, however, entirely discursive. It is our contention that the different spaces in which these interpretations and discussions took place, and the various technologies which underpin and co-create those digital environments, significantly shape the resulting digital artefact, and cannot be reduced entirely to human intention.

Mostly prominently perhaps, is the algorithmic process that produces the wordcloud, in this case from web service 'Tagxedo,' performing a number of automatic processes upon the source text. This includes the variables 'emphasis' and 'tightness'; the former controlling the increase in font size in relation to the frequency of words encountered, while the latter regulates how the collection of words is arranged within the over-arching shape (Tagxedo, no date). Both these variables can be customised by the user, but also impose automated and pre-programmed conditions upon the resulting image, the effects of which cannot be attributed exclusively to the programmer, the end-user, or the code itself. Furthermore, the Tagxedo algorithm executes a number of processes in which the end user has no control; including the removal of pre-determined function words (is, at, the, etc.) and the combining of different tenses, known as 'stemming.' Additionally, the heart shape which this digital artefact appears to resemble is also produced algorithmically from an encoded template; a process which determines the position and proximity of words. The intermingling words and algorithms of 'World Builder: a crowd-sourced tag heart' thus embody the simultaneity of material processes and symbolic representation. It thus expresses a non-dualistic and relational ordering of the world, in which 'matter is not dialectically opposed to culture, nor to technical mediation, but continuous with them' (Braidotti 2013, p. 35). Codes and discursive meanings operate concurrently to produce the artefact. 'Social' and 'material' do not remain distinct dimensions that interact, rather words change how the algorithms operate, and they in turn alter size and order of the text, and thus the discursive interpretations that might be construed.

The 'final state' of this digital artefact also, we suggest, troubles the prevailing notions of digital practice. 'World Builder: a crowd-sourced tag heart' 'exists' in a number of web locations: the Tagxedo website, the Flickr photo-sharing service, and the student created digital artefact 'Wallwisher' (a collection of final assignment submissions see Figure 1), spaces which re-contextualise the work, entangling the 
linguistic and visual features of the piece with different themes and settings. Moreover, the Tagxedo output is interactive; each word serves as a hyperlink to a Google search based on that term. Thus the artefact can be viewed, not simply as a contained piece of work with specific and fixed properties, but as also involving relations with exterior spaces, constituting the virtual capacities of an assemblage (DeLanda 2006). Therefore, to abridge the complex processes that have produced this image to the intentions of a lone student and an absolute list of competencies would seem to disregard the array of socio-material and relational conditions necessary for it to have come together. Rather than representing knowledge about the film or the course, the artefact performs knowledge through relational interplay between the participants, their discussions, and the abstracted software algorithms of the web. We will return to this point in the next section. Furthermore, to consider 'World Builder: a crowd-sourced tag heart' as a confined representation of knowledge would appear to discount its capacity for connection and re-appropriation, and ultimately the instability of the digital.

Our second example - the digital artefact 'E-learning and Human 3.0' - was created with 'Videoscribe' presentation software, rendered as a video and uploaded to You Tube (Figure 3). It exemplifies a number of layered processes that are embedded in digital systems, ordered through multiple relations and contingencies, and typical of a range of EDCMOOC multimodal practices. The presentation consists of text and images, accompanied by the animated reproduction of a hand pre-programmed by the software to appear as if it is inscribing the words and sketching the visuals. Such presentations are created by inputting and positioning text, choosing from a library of pre-set images, and selecting a preferred limb with accompanying writing implement for the animation (see Figure 4). Once the arrangement is complete, the software provides a 'play' feature which will animate each element in the presentation

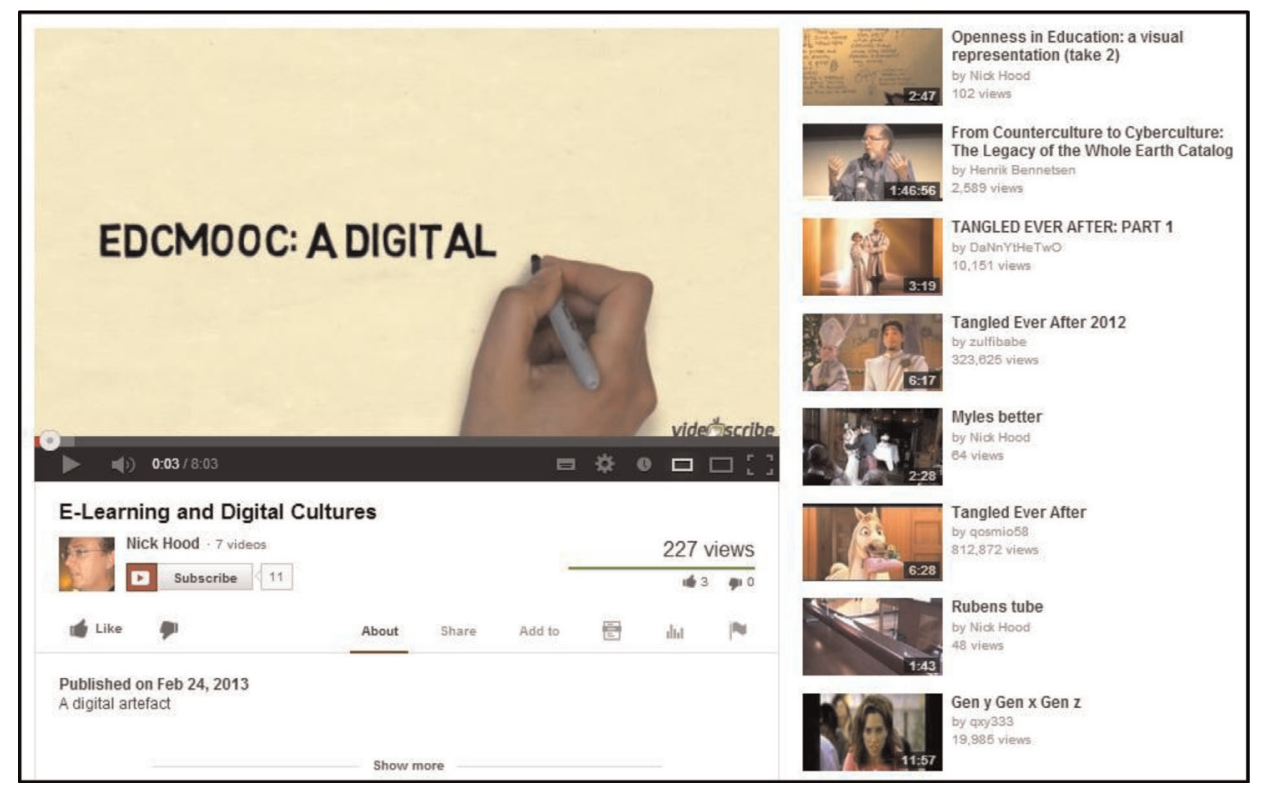

Figure 3. 'E-learning and Human 3.0' by Nick Hood, digital artefact created for the EDCMOOC: http://www.youtube.com/watch?v=6JPq60Hd8CE 


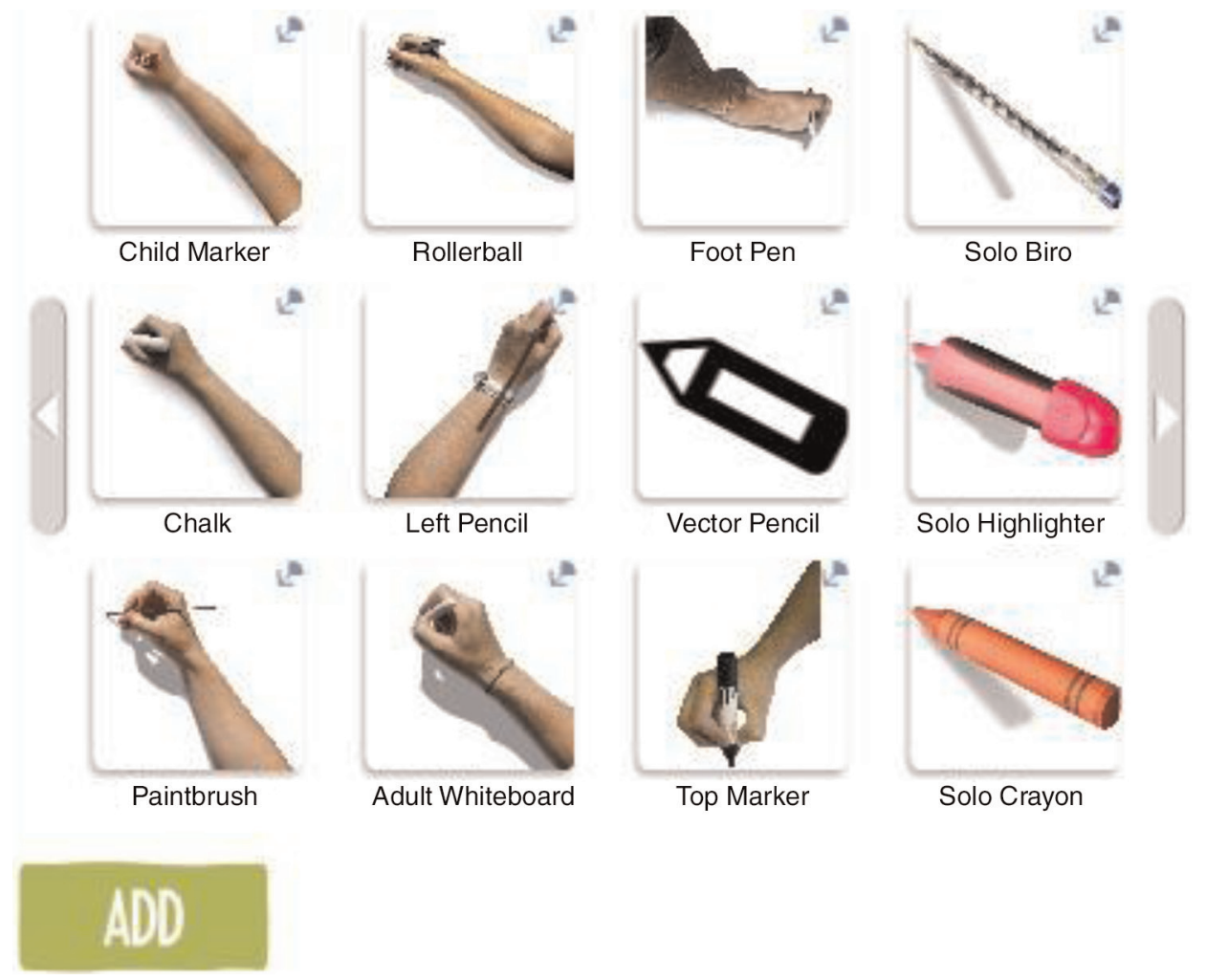

Figure 4. Limb and writing implement choices from the 'Videoscribe' presentation software.

by making it appear to emerge gradually from the movement of the image of the writing hand.

Videoscribe represents an archetypal 'black boxing' of digital media effects and processes (Fenwick and Edwards 2010). Rather than presiding over each and every detail of the process, users select from a number of predetermined visualisations and animations, an arrangement which reduces author control. Videoscribe thus illustrates a tension between software accessibility and usability on the one hand, and openness and user agency on the other. The more polished the user interfaces, the more sophisticated and inaccessible the underlying code (Edwards and Carmichael 2012). The focus on interface usability is indicative of perceiving software in terms of a 'tool' or 'application' that can accomplish particular tasks, such that the use of technology becomes 'seamless and unremarkable' (Edwards and Carmichael 2012, p. 5). The inconspicuous educational activity produced here is a 'whiteboard-style' presentation, complete with the writing hand of the teacher and accompanied with a pre-recorded voiceover. However, a socio-material approach might help us to consider this, not simply as an expression of competence in the digital domain, reproducing classroom practice through multimodal technologies, but rather as composite ordering of space, absence and presence.

The sound of the voice and the moving image of the hand are multimodal embellishments which attempt to centre a human author as present and in command of the learning activity, while the presentation format itself draws upon notions of established classroom spaces and a linear lecture-type arrangement. However, the 
supposed corporeality of the teacher here is the result of encoding practices which conceal non-human agencies. This is most notable in the image of the hand which has not only been selected from a library of pre-set images, thus deriving from a different body entirely from that of the 'author' (Figure 4), but is also animated with software that precedes the text and images that it might render in the final piece. The body of the teacher is thus performed here through an assemblage of multiple bodies, codes and texts. The symbolic qualities of the teacher are not negated in this socio-material reading; rather they are shown to be simultaneously and co-constitutively nonsymbolic, and non-human.

The apparently straightforward emulation of classroom space offered by the 'E-learning and Human 3.0' is equally problematic when we consider the specific conditions of its location in You Tube (see Figure 3), which we consider to be an integral part of the spatial ordering of the artefact itself. Of particular relevance here are the 'recommended videos' which border the artefact, and the comments which cluster below. Recommended videos are determined by a broad range of data, including meta-data associated with each video, a logged-in user's previous activity, as well as the viewing activity of others (Davidson et al. 2010). Not having access to the specific algorithms and codes that are here being considered in terms of the nonhuman or material may be problematic for an in-depth analysis, a criticism Mutch has levelled at socio-materialism (2013). However, the point is not to define exact processes or modes of activity, but rather to acknowledge that agency cannot be reduced to either wholly human or non-human foundations. We can say that this is a complex and on-going amalgamation of human intention, text and algorithmic process which persistently restructures the YouTube page, and thus the setting in which the artefact is situated, and the spatial ordering of which it is a part. As we can see in Figure 3, this range of factors has produced a diverse mix of educational and internet-related content, both personal and professional videos uploaded by the author, and animated films from Disney. This radically re-contextualises the conventional classroom space offered by the 'E-learning and Human 3.0' presentation. This seems to be a space in which knowledge, author, and absent You Tube user are enacted simultaneously.

\section{What do these examples mean for digital literacy?}

Underpinned by a philosophical approach that seeks to work against the dualisms that structure and maintain established orientations of representationalist thinking, we suggest that socio-material theory has profound implications for the concept of digital literacy. Representationalism can be understood as 'the notion that the world is brought into being by humans who go about knowing and naming observationindependent objects with attributes' (Scott and Orlikowski 2013, p. 78). Such designed entities are taken to be foundational; naturalised categorisations and distinct objects with which we interact, a process which masks the relational practices that have come together to produce, and maintain the representation. Thus a representationalist epistemology is suggested to produce the subject/object distinction, within which 'the world is made up of objects 'out-there' that we try to know 'in-here' within the knowing subject' (Edwards 2010, p. 10). Therefore, in suggesting that socio-material theory be brought to the field of digital literacy, two crucial and interrelated, but not unproblematic, premises need to be explored: representationalism and anthropocentrism. 


\section{J. Knox and S. Bayne}

As illustrated in the 'World Builder: a crowd-sourced tag heart' example earlier, the complex and distributed factors which produced the word-cloud problematize the notion of representational knowledge. Can this digital artefact be measured in terms of how accurately it signifies the ideas intended by its creator, particularly as we have tried to question the notion of the autonomous author? Simply to consider what kind of 'literacy' is exemplified by 'World Builder: a crowd-sourced tag heart' would appear to dismiss the rich, situated socio-material practices which performed and instantiated the particular arrangement of words. Constituents of this enactment, as we have seen, are software codes and algorithms, which we consider to be independent actors (Edwards and Carmichael 2012). With reference to the website TripAdvisor, Scott and Orlikowski (2013) state that rather than viewing the algorithm as 'as a mirror of conscious socio-technical choices - a snapshot produced from a passive collage of human intention - we have reframed it as a highly specific, active, partial, generative (performative) engine involved in re-making the world of travel' (p. 78). The potential for exhaustive iterations encoded in the algorithm cannot be reduced to the foundational intentions of the programmer or be determined as the exclusive agency of artificial intelligence. Its inclusion blurs the simple causal relationship and thus the identification of representative meaning. In other words, where the production of knowledge is distributed and relational, the human mind would only be a constituent part of such an entanglement, and whatever its cognitive make-up might be, we could not define it as being identical with the wider socio-material assemblage. It is fruitless, we suggest, to attempt to disentangle 'World Builder: a crowd-sourced tag heart' from the processes of its production. Yet, the focus on defined competencies would appear to seek this kind of separation; defining digital literacy as a set of skills which can be measured in the production of digital artefacts through a transparent and anthropocentric meaning-making process.

As we have seen in the 'E-Learning and Human 3.0' digital artefact, educational space, and the presence and absence of the teacher might be considered the enactments of complex socio-material orderings, rather than naturalised or anterior realities. The intentional presence of a single human educator would be difficult to pinpoint, distributed as it is among encoded bodily characteristics and automated software processes. Such an analysis is productive because it encourages a critical rather than instrumental view of technology, urging deeper considerations of the political enactments that software and code are already implicated in. For example, all the default choices for animation appear to depict limbs with white skin (see Figure 4), calling into question the pedagogical and political neutrality of such educational 'tools'. Digital literacy thus needs to contend with the cultural norms and beliefs that are already encoded into the technologies we use, and are performed by them in educational activity, rather than defaulting to the rhetoric of unproblematic empowerment, emancipation, or indeed professional competency through the utilisation of technology. A shift in perspective toward enacted events, rather than simple representations of knowledge, help to uncover the multiple agents, purposes and rationales that collide through educational activity. Both these artefacts suggest that digital literacy is not just a mix of different literacies (Oliver 2012; Thomas et al. 2007), but a meshwork of agencies. To be digitally literate, therefore, is to have already engaged in entanglements which decentre the autonomous learning subject. This blurs further dualisms, not just academic/cultural (Carpenter 2009), but also social and material. Ultimately, to engage in the production of multimodal 
digital artefacts is to involve oneself in complex entanglements of contexts, cultures and technologies, of which the so-called human 'producer' is merely a constituent part.

The discussion of non-human agency is not foregrounded here for the purposes of suggesting that such processes can be examined, isolated and comprehended as exclusively 'material,' and thus allowing the discerning researcher to determine which precise elements of digital practice derive from human will. This would be to miss the point entirely. Rather, we propose that digital literacy be perceived as a practice always and already enmeshed in composite socio-material assemblages, in which human involvement needs to be recognised as partial, irreducible and sometimes modest. Edwards and Carmichael (2012) call into question the emancipatory ideal of utilising digital technologies for creative meaning-making practices, rather than simply consuming media. Digital literacy as liberation is premised on ideas of mastery, in which the user is re-centred and digital systems become subservient to human intention and their capacity to act discounted. We suggest that digital literacy can productively move forward by engaging with theoretical areas which de-centre human intention, and acknowledge the irreducibility and entanglement of sociomaterial factors in educational activity.

\section{Conclusions}

As we have seen, digital literacies are predominantly understood either as competencies or as sociocultural practices. We can perhaps view the habitual calls for culture change, institutional transformation, and individual 'future proofing' through skillsbased training as a tacit acknowledgement of the ways in which human beings begin to lose the sense of stability and authority when educational activity enters the digital domain. Digital literacy, when considered as merely 'up-skilling,' is the attempt to regain supremacy and mastery in a territory devoid of the comforts of established disciplines and authoritative texts. Thus our focus here has been less on the scholarly merit of using digital media - a position which seems to preserve an epistemological orientation that privileges the human being - and more concerned with how to situate it as a practice, nevertheless a practice with pedagogical implications.

Decentring the individual is to move beyond notions of training and skills development, and to step towards viewing multimodal practices as socio-material enactments. Thus digital literacy can be theorised as a contingent, specific and relational practice. The creation of digital artefacts in the EDCMOOC constituted just such an enactment of digital literacy; complex in the sense that what was produced was drawn from a vast array of human and non-human agencies and contexts, but also specific in that all were responsive to and concentrated upon the course activities and content. Thus, rather than thinking of digital literacy as a set of obligatory skills derived from the demands of future labour, it becomes the collective enactment of knowledge in response to both distributed and centralised contingencies. The pedagogical value of this perspective would be to acknowledge the situated practices of digital work; to recognise the broader social and cultural contingencies that shape what we produce in educational contexts, as well as the embedded algorithms and codes of digital media that co-create the artefacts of the web. 


\section{J. Knox and S. Bayne}

\section{References}

Bawden, D. (2008) 'Origins and concepts of digital literacy', in Digital Literacies: Concepts, Policies and Practices, eds C. Lankshear \& M. Knobel, Peter Lang, Oxford, pp. 17-32.

Beetham, H., McGill, L. \& Littlejohn, A. (2009) Thriving in the 21st century: learning literacies for the digital age (LLiDA project), [online] Available at: http://www.jisc.ac.uk/ media/documents/projects/liidareportjune2009.pdf

Braidotti, R. (2013) The Posthuman, Polity Press, Cambridge.

Branit, B. (2007) World builder, [online] Available at: http://youtu.be/VzFpg271sm8

Carpenter, R. (2009) 'Boundary negotiations: electronic environments as interface', Computers and Composition, vol. 26, no. 3, pp. 138-148.

Davidson, J., et al. (2010) 'The YouTube video recommendation system', in Proceedings of the Fourth ACM Conference on Recommender Systems. pp. 293-296, [online] Available at: http://dl.acm.org/citation.cfm?id=1864770

DeLanda, M. (2006) A New Philosophy of Society: Assemblage Theory and Social Complexity, Continuum, London.

Edwards, R. (2010) 'The end of lifelong learning: a post-human condition?' Studies in the Education of Adults, vol. 42, no. 1, pp. 5-17.

Edwards, R. \& Carmichael, P. (2012) 'Secret codes: the hidden curriculum of semantic web technologies', Discourse: Studies in the Cultural Politics of Education, vol. 33, no. 4, pp. $1-16$.

Fenwick, T. \& Edwards, R. (2010) Actor-Network Theory in Education, Routledge, Abingdon.

Fenwick, T., Edwards, R. \& Sawchuk, P. (2011) Emerging Approaches to Educational Research: Tracing the sociomaterial, Routledge, Abingdon.

Fenwick, T. \& Landri, P. (2012) 'Materialities, textures and pedagogies: socio-material assemblages in education', Pedagogy, Culture and Society, vol. 20, no. 1, pp. 1-7.

Friesen, N. \& Hamilton, E. (2010) 'Online education: the view from technology studies', in Proceedings for the Technological Learning and Thinking Conference: Culture, Design, Sustainability, Human Ingenuity, [online] Available at: http://m1.cust.educ.ubc.ca/conference/ index.php/TLT/2010/schedConf/presentations

Goodfellow, R. (2011) 'Literacy, literacies and the digital in higher education', Teaching in Higher Education, vol. 16, no. 1, pp. 131-144.

Goodfellow, R. \& Lea, M. (2007) Challenging E-learning in the University: A Literacies Perspective, Open University Press, Maidenhead.

Goodfellow, R. \& Lea, M. (2009) 'Academic Literacies in the Digital University', in Literacy in the Digital University: The Relation of New Media Practices to Traditional Literacy Practices in the Academy and the Professions, Edinburgh University, [online] Available at: http://oro.open.ac.uk/25393/2/

Hannon, J. (2013) 'Incommensurate practices: sociomaterial entanglements of learning technology implementation', Journal of Computer Assisted Learning, vol. 29, no. 2, pp. $168-178$.

JISC. (2013). Developing students' digital literacy to give them the best chance of success, [online] Available at: http://www.jisc.ac.uk/supportingyourinstitution/studentjourney/digilit.aspx

Lankshear, C. \& Knobel, M. (2008) Digital Literacies: Concepts, Policies and Practices, Peter Lang, Oxford.

Mutch, A. (2013) 'Sociomateriality - taking the wrong turning?' Information and Organization, vol. 23 , no. 1 , pp. $28-40$.

Oliver, M. (2012) 'Learning with technology as coordinated sociomaterial practice: digital literacies as a site of praxiological study', in 8th International Conference on Networked Learning 2012, eds. V. Hodgson, et al., pp. 440-447, [online] Available at: http://www. lancaster.ac.uk/fss/organisations/netlc/past/nlc2012/info/confpapers.html

Scott, S. V. \& Orlikowski, W. J. (2013) 'Sociomateriality - taking the wrong turning? A response to Mutch', Information and Organization, vol. 23, no. 2, pp. 77-80.

Tagxedo (no date) Frequently Asked Questions, [online] Available at http://www.tagxedo.com/ faq.html

Thomas, S., et al. (2007) 'Transliteracy: crossing divides', First Monday, vol. 12, no. 12, [online] Available at: http://firstmonday.org/ojs/index.php/fm/article/view/2060/1908

Ulmer, G. (2003) Internet Invention: From Literacy to Electracy, Longman, New York. 